\title{
Development of electrochemical biosensors by e-beam lithography for medical diagnostics
}

\author{
Francesca Virgilio $^{\mathrm{a}, *}$, Mauro Prasciolu ${ }^{\mathrm{a}, 1}$, Paolo Ugo ${ }^{\mathrm{b}}$, Massimo Tormen ${ }^{\mathrm{a}}$ \\ a IOM-CNR, TASC Laboratory, Area Science Park - Basovizza, S.S 14 Km 163.5, I-34149 Trieste, Italy \\ ${ }^{\mathrm{b}}$ Department of Molecular Sciences and Nanosystems, University Ca' Foscari of Venice, Santa Marta 2137, 30123 Venice, Italy
}

\section{A R T I C L E I N F O}

\section{Article history:}

Available online 24 February 2013

\section{Keywords:}

Nanoelectrode arrays

Boron-doped diamond

Electron beam lithography

Cyclic voltammetry

DNA immobilization

\begin{abstract}
A B S T R A C T
In this work the fabrication and characterization of boron-doped diamond (BDD) nanoelectrode arrays are discussed. The use of boron-doped diamond electrodes is very attractive due to advantageous properties including high reproducibility, stability, and robustness under extreme conditions, where conventional electrode materials may undergo severe erosion. BDD electrodes have also proved to be very useful because they show an extremely wide potential window in aqueous solutions without oxidation of the electrode itself. This allows electrochemical detection, at tiny background currents, of a number of substances that oxidize at very positive potentials, where other electrodic materials are not suitable. BDD based NEAs were prepared using $\mathrm{Si}\langle 100\rangle$ substrates coated with a layer of Boron doped diamond as macroelectrode. NEAs were obtained by creating an array of nanoholes by electron beam lithography (EBL) in a thin film of polycarbonate deposited on top of the macroelectrode. This approach leads to the formation of recessed nanoelectrodes. The parameters for using polycarbonate as a novel electron beam resist have been optimized and successfully used for fabrication of NEAs. The most interesting properties of this polymer for nanofabrication purposes are the high lithographic contrast, which allows the creation of structures of dimensions less than $100 \mathrm{~nm}$; chemical stability, which guarantees a long-term use in electrochemical solutions and the possibility of functionalization with biological molecules (DNA and proteins). NEAs have been characterized with cyclic voltammetry and have provided voltammetric signals controlled by pure radial diffusion. The low background current of BDD added to the properties of NEAs indicate that this system can be applied for the development of sensors with high sensitivity. Polycarbonate surface of NEAs was successfully functionalized with small ss-DNA sequence, confirming the possibility of exploiting these systems as diagnostic biosensors.
\end{abstract}

(c) 2013 Elsevier B.V. All rights reserved.

\section{Introduction}

Chemical and biochemical sensors are devices able to provide information concerning the presence of molecules and biomolecules through the measurement of a signal (mainly, optical or electrical) generated by a transducer in intimate contact with a (bio-) recognition layer [1]. In electrochemical sensors, a change in the redox state of the biorecognition/analyte system related to the biorecognition event, produces a change in an electrochemical quantity, which can be monitored by classical electroanalytical techniques. Electrochemical systems can be miniaturized below the micrometer scale, using ordered arrays of nanoelectrodes (NEAs) [2,3]. A NEA is made by a very large number of very small

\footnotetext{
* Corresponding author. Tel.: +39 040375 8414; fax: +39 040226767.

E-mail addresses: virgilio@iom.cnr.it (F. Virgilio), mauro.prasciolu@desy.de (M. Prasciolu), ugo@unive.it (P. Ugo), tormen@iom.cnr.it (M. Tormen).

1 DESY, Photon Science, FS-ML Group, Notkestr. 85, Geb. 99/Room 3.49, 22609 Hamburg, Germany. Tel.: +49 408998 5782; fax: +49 4089941958.
}

ultramicroelectrodes separated by an electrical insulator, with density between $10^{6}$ and $10^{8}$ electrodes $/ \mathrm{cm}^{2}$. The electrochemical characteristics that distinguish nanoelectrodes arrays from conventional macro (mm-sized) or even ultramicro ( $\mu \mathrm{m}$-sized) electrodes are dramatic lowering of double-layer charging (capacitive) currents (that are the largest component of the noise, or more properly the background signal of voltammetric measurements) $[4,5]$ and extreme sensitivity to the kinetics of the charge transfer process [6], which means capability to measure very high charge transfer rate constants [7]. Moreover, NEA can exhibit different diffusion regimes. According to the model proposed by Guo and Lindner [8], diffusion regimes occurring to an array of microelectrodes can be divided into five categories:

(I) planar diffusion over each microelectrode;

(II) mixed diffusion over each microelectrode (transition between planar and hemispherical diffusion);

(III) diffusion over each hemispherical microelectrode; 
(IV) diffusion mixed with onset of overlap of diffusion layers;

(V) planar diffusion over the entire array.

The characteristics of the time-dependent diffusion profile of electrode arrays depend primarily on the relative inter-electrode spacing, $d / r$ (where $d$ is the center-to-center distance between electrodes and $r$ is the radius of nanoelectrode), as well as on the timescale of the experiment. In the case of widely spaced array (with a large $d / r$ ratio), diffusion of the electroactive species to each individual electrode remains independent of all others for the duration of the experiment, and the array behave in the same manner as multiple single electrodes in parallel [9]. This is the regime that gives the higher Faradic current, therefore this is the regime of choice for obtaining the maximum improvement of detection limits when there are no constraints in increasing the distance between the nanoelectrodes.

The ability of NEA to furnish well resolved cyclic voltamograms can allow to develop sensitive methods for trace determination of redox species characterized by fast electron transfer kinetics. Moreover, the lowering of the background noise (related to the capacitive current) in an electrochemical determination improves not only the detection limit but also the precision of the determination [10].

The aim of this work is to combine the properties of arrays of nano-electrodes with the advantages of boron-doped diamond (BDD) as electrodic material. The use of boron-doped diamond electrodes is very attractive due to its high stability and robustness under extreme conditions, where conventional electrode materials may undergo severe erosion. BDD electrodes have also proved to be very useful because they show an extremely wide potential window in aqueous solutions without oxidation of the electrode itself. This allows electrochemical detection, at tiny background currents, of a number of substances that oxidize at very positive potentials, where other electrodic materials are not suitable [11].

Concerning the dielectric film used for the isolation of nanoelectrodes, polycarbonate has been selected knowing that this polymer was successfully used to produce Nanoelectrodes Ensembles (NEEs) based on track etched membranes [4,5,12]. In particular this polymer shows satisfactory properties of chemical stability in common electrochemical solutions employed in voltammetric measurements and the possibility of biochemical functionalization. In typical schemes used to obtain electrochemical biosensors, a biorecognition layer is directly immobilized on the electrode surface and the signal is produced by exchange of electrons between this layer and the underlying electrode. However, in case of nanoelectrodes, is more convenient to immobilize the biological probes on the polymer surrounding the electrodes and not on the nanoelectrodes themselves. In such a design, transducer and biorecogniton layer are not overlapped, but integrated in strict proximity $[13,14]$. The use of polycarbonate as a resist was first demonstrated for the fabrication of NEAs by e-beam lithography on gold macroelectrodes [15]. Attempts of using PMMA for the same purpose did not provide completely satisfactory results [16], in particular concerning the stability and reproducibility in electrolyte solutions.

\section{Materials and methods}

\subsection{Materials and instruments}

Polycarbonate solutions ( $2 \%$ and $3 \% \mathrm{w} / \mathrm{v}$ ) were obtained by dissolution of solid Polycarbonate Makrolon (Bayer Sheet Europe) in cyclopentanone. The salt (ferrocenylmethyl) trimethylammonium hexafluorophosphate $\left(\mathrm{FA}^{+} \mathrm{PF}_{6}^{-}\right)$was prepared by metathesis of the (ferrocenylmethyl)trimethylammonium iodide (Alfa Aesar) with potassium hexafluorophosphate 99\% (Alfa Aesar). Amino-end DNA (acD1) labeled with fluorescein was purchased from TAG Copenhagen $\mathrm{A} / \mathrm{S}$ and had the following sequences:

acD1: 5'amino CX-CTT ATC GCT TTA TGA CCG GAC C-fluorescein-3'. Other chemicals were purchase from Sigma-Aldrich, unless otherwise stated, and were used as received. Purified water was obtained by a Milli-RO plus Milli-Q (Millipore) system.

EBL was performed using a Zeiss $1540 \mathrm{XB}$ cross beam instrument equipped with RAITH ELPHY nanolithography system. Contact angles measurements were obtained by optical tensiometry with a KRÜSS contact angle measuring instrument (GmbH, Germany). Inductively Coupled Plasma treatments were performed using a Surface Technology Systems ICP Instrument. Fluorescent images were taken with a commercial inverted microscope Nikon Eclipse.

Electrochemical measurements were carried out at room temperature in a three-electrode cell with a platinum coil counter electrode and an $\mathrm{Ag} / \mathrm{AgCl}$ ( $\mathrm{KCl}$ satured) reference electrode, connected to a CHI Model 660B potentiostat (CHIJ Cambria Scientific, UK) controlled by its own software.

\subsection{Nanoelectrodes arrays fabrication}

For the fabrication of NEAs, $\mathrm{Si}\langle 100\rangle$ substrates coated with a $400 \mathrm{~nm}$ thick layer of BDD (Adamant Technologies SA), were used as macroelectrodes. Films of polycarbonate (PC) were spin coated on the BDD surface at $2000 \mathrm{rpm}$ and baked at $180^{\circ} \mathrm{C}$ for $30 \mathrm{~min}$. The resulting average film thickness (measured with a Dektak Profilometer) for samples prepared from PC solutions at 2\% and 3\% were $22 \mathrm{~nm}$ and $87 \mathrm{~nm}$, respectively. The pattern of the NEA was exposed on the samples by a focused electron beam at $30 \mathrm{kV}$ and developed $1 \mathrm{~min}$ in $\mathrm{NaOH} 5 \mathrm{M}$ at $35^{\circ} \mathrm{C}$. The opened areas were cleaned from residual of resist by oxygen plasma at $4 \mathrm{mT}$ pressure, applying $200 \mathrm{~W}$ and $10 \mathrm{~W}$ RF power to the coil and to the platen $\left(8^{\prime \prime}\right)$ respectively, resulting in a bias of $30 \mathrm{~V}$. Different durations of plasma treatments were tested. The etching rate of polycarbonate was $140 \mathrm{~nm} / \mathrm{min}(23 \AA / \mathrm{s})$. This step allows also the activation of PC suface by formation of oxygenated groups. The effect of oxygen plasma on PC surface was investigated by contact angle measurement.

\subsection{Surface functionalization}

Prior to the immobilization of the oligonucleotide probes on the polycarbonate surface, NEAs were incubated in a $0.1 \mathrm{M}$ HEPES buffer solution ( $\mathrm{pH} 7.5$ ) containing $0.5 \mathrm{mM} \mathrm{N}$-ethyl- $\mathrm{N}^{\prime}$-(3-dimethylaminopropyl) carbodiimide hydrochloride (EDC), and $0.8 \mathrm{mM}$ $\mathrm{N}$-hydroxysulfosuccinimide sodium salt (sulfo-NHS), for $20 \mathrm{~min}$ under stirring at room temperature. This step is necessary in order to activate the carboxylic groups present on the PC surface of NEAs enabling the reaction of - $\mathrm{COOH}$ groups with the amino group of the ss-DNA. The intermediate ester obtained by reaction between EDC and carboxylate groups is stabilized by the addition of the succinimide derivative, thus increasing the efficiency of the coupling reaction with the amino-ss DNA (acD1). The functionalization was done transferring the electrodes into a $0.4 \mu \mathrm{M}$ acD1 solution in $0.1 \mathrm{M}$ HEPES buffer solution ( $\mathrm{pH} 7.5$ ) and incubated for 2 h under stirring, at $37^{\circ} \mathrm{C}$. The NEAs were then rinsed with buffer and treated with a $20 \mathrm{mM}$ ethanolamine in $0.01 \mathrm{M}$ PBS solution ( $\mathrm{pH} 7.4$ ) in order to deactivate the remaining NHS-activated carboxylic groups onto the polymeric surface. The reaction was allowed to proceed for 30 min at room temperature, under stirring. To evaluate the effective immobilization of the probe on the surface, the electrodes were observed with a fluorescence microscope using a magnification $60 \times$ with $\mathrm{H}_{2} \mathrm{O}$ differential interference contrast $1.5 \times$. 


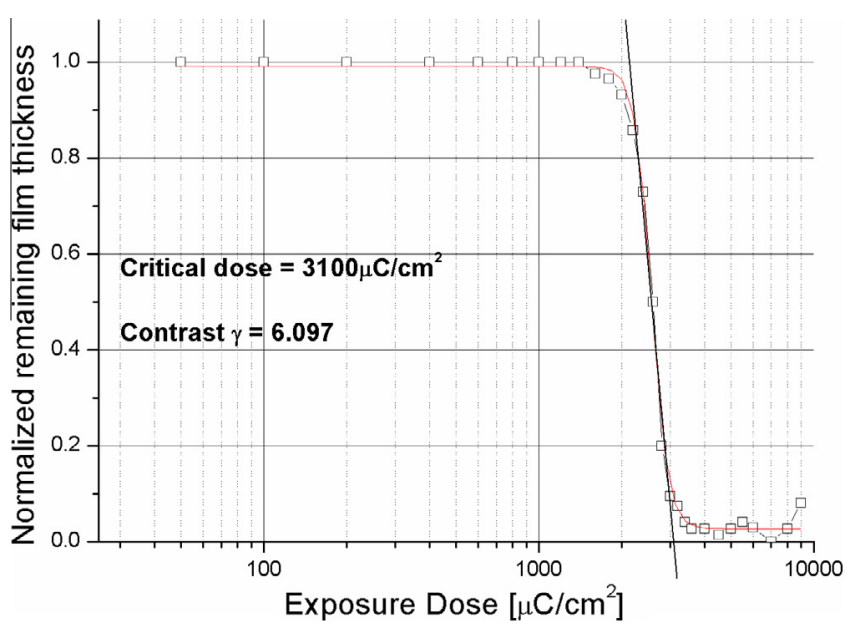

Fig. 1. NRT curve for polycarbonate Makrolon $2 \%$ at $30 \mathrm{kV}$.

\section{Result and discussion}

\subsection{Parameters optimization}

Contrast curves for PC as positive electron-beam resist were obtained by exposing a simple test pattern (dose matrix with an array of $50 \mu \mathrm{m} \times 100 \mu \mathrm{m}$ rectangles) on $125 \mathrm{~nm}$ thick film of PC deposited thick $\mathrm{Cr} / \mathrm{Au}(10 / 20 \mathrm{~nm})$ baseplating on $\mathrm{Si}\langle 100\rangle$. Exposure doses ranged between $50 \mu \mathrm{C} / \mathrm{cm}^{2}$ and $11,000 \mu \mathrm{C} / \mathrm{cm}^{2}$ and $30 \mathrm{keV}$ electron energy was used. The resulting Normalized Remaining film Thickness (NRT) curve is shown in Fig. 1.

The contrast $\gamma$ was calculated according to the following equation:

$\gamma=\left[\log _{10}\left(\frac{D_{0}}{D_{1}}\right)\right]^{-1}$

where $D_{0}$ is the extrapolated critical dose necessary to completely remove the resist, and $D_{1}$ is the extrapolated dose at which the developer first begins to attack the irradiated film.

Next, the optimal dose for a high resolution patterning on BDD substrate was tested, writing a pattern $4 \times 4$ of holes with dose range between $2500 \mu \mathrm{C} / \mathrm{cm}^{2}$ and $10,000 \mu \mathrm{C} / \mathrm{cm}^{2}$. The optimal dose for development performed at $35{ }^{\circ} \mathrm{C}$ in $\mathrm{NaOH} 5 \mathrm{M}$ resulted to be $8000 \mu \mathrm{C} / \mathrm{cm}^{2}$. Using the optimized parameters, NEAs containing $1.16 * 10^{4}$ nanoelectrode has been obtained, with a density of $1.3 * 10^{7}$ nanoelectrodes $/ \mathrm{cm}^{2}$.

Fig. 2 shows SEM image of a section of obtained array and the magnification of one of the nanoelectrode, where can be seen the underlying nanocrystalline BDD layer:

The NEAs were treated with different time of $\mathrm{O}_{2}$ plasma and the degree of surface modification was assessed by contact angle measurements. It was observed that the contact angle varied from $97^{\circ}$ for untreated PC to $42^{\circ}$ for PC treated for $8 \mathrm{~s}$, indicating an increase in the hydrophilicity of the surface, due to an increase of oxygenated groups.

\subsection{Electrochemical characterization}

The fabricated NEAs were tested electrochemically using (Ferrocenylmethyl) trimethylammonium hexafluorophosphate $\left(\mathrm{FA}^{+} \mathrm{PF}_{6}^{-}\right) 10^{-3} \mathrm{M}$ as reversible redox probe and $\mathrm{NaNO}_{3} 0.5 \mathrm{M}$ as supporting electrolyte. Cyclic voltammograms shown in Fig. 3 were recorded at different scan rates (from 5 to $500 \mathrm{mV} / \mathrm{s}$ ).

The main effect of the plasma treatment is to fully remove residues of polycarbonate from the diamond surface in the holes, which results in an increase of the electrode resistance. From Fig. 3(a)-(c) it can be noticed that the treatment with $\mathrm{O}_{2}$ plasma effectively improves the voltammetric signals and that in case (c) the voltammetric patterns acquire a sigmoidal shape over a relatively broad range of scan rates, indicating that under these conditions the diffusion regime is purely radial. From Fig. 3(d)-(f), that refer to NEAs obtained from PC3\% and therefore more recessed, it can be noticed that for scan rates $\geqslant 50 \mathrm{mV} / \mathrm{s}$ a transition from sigmoidal to mixed shaped CVs occurs.

The steady state limiting current was calculated extending to our NEAs the model proposed by Bond et al. [17] for a single recessed nanoelectrode:

$I_{l i m}=\frac{4 \pi n F C D r^{2}}{4 l+\pi r}$

where $n$ is the number of electrons exchanged, $F$ is the Faraday constant, $C$ is the analyte concentration, $D$ is the diffusion coefficient, $r$ is the hole radius and $l$ is the recession depth. The recession depth measured with a Dektak Profilometer resulted to be $22 \mathrm{~nm}$ for NEAs obtained from PC2\% and $87 \mathrm{~nm}$ for NEAs obtained from PC3\%, and,

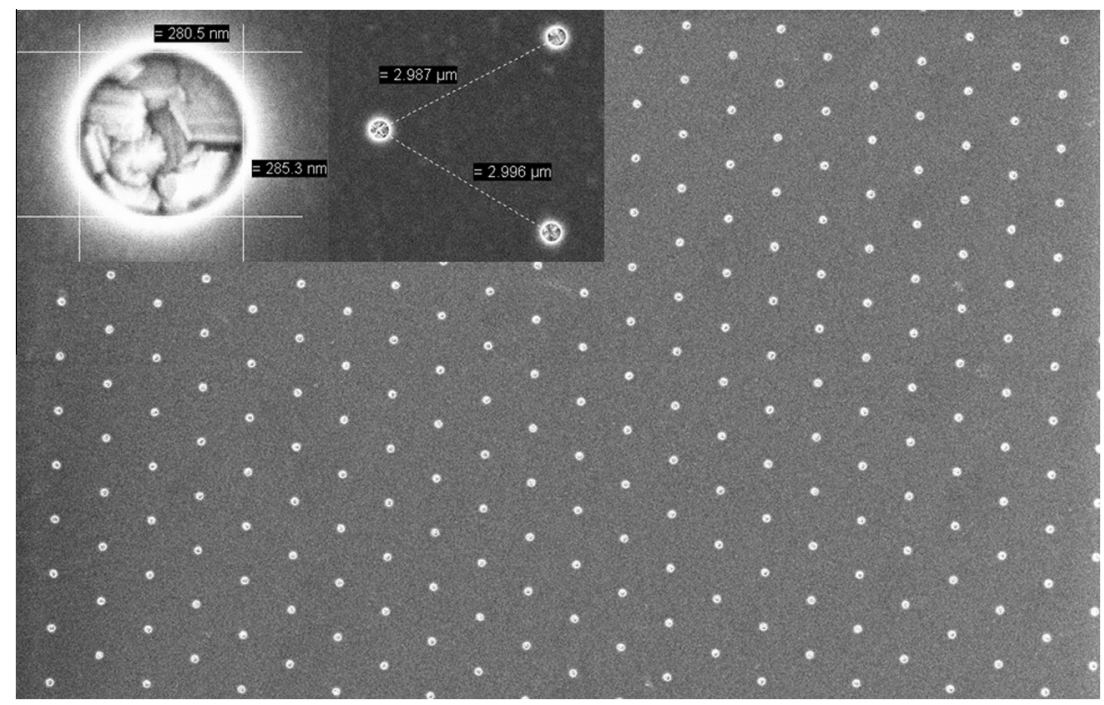

Fig. 2. SEM images of NEA with dots of average radius of $140 \mathrm{~nm}$ and interspacing distance of $3 \mu \mathrm{m}$. 

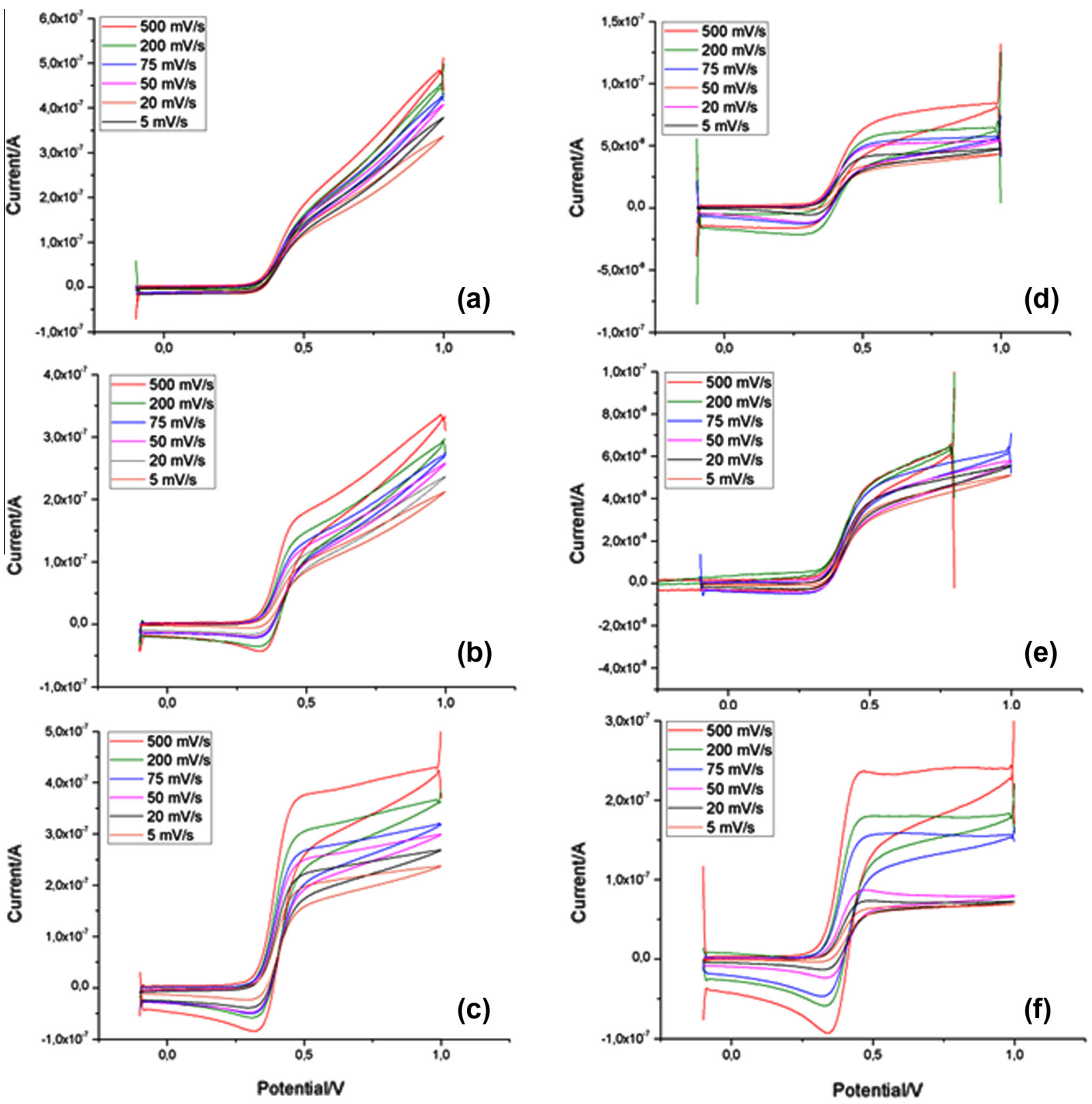

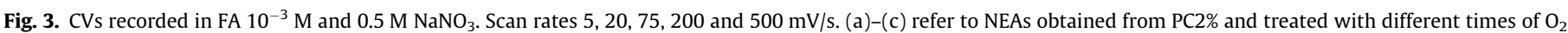
plasma (respectively, 0, 2 and $4 \mathrm{~s}$ ); (d)-(f) refer to NEAs obtained from PC3\% and treated with different times of $\mathrm{O}_{2}$ plasma (respectively, 0,4 and $8 \mathrm{~s}$ ).

as expected from the theoretical model, with the increasing of the recession depth corresponds a decreasing of the limiting current.

Calculated and measured limiting currents are reported in Table 1:

Table 1

Comparison between experimental and calculated limiting current for NEAs obtained from $\mathrm{PC} \%$ and $\mathrm{PC} 3 \%$.

\begin{tabular}{llllll}
\hline \multirow{2}{*}{$\begin{array}{l}\mathrm{O}_{2} \text { Plasma time } \\
(\mathrm{sec})\end{array}$} & \multicolumn{2}{l}{$\mathrm{PC} \%$} & & \multicolumn{2}{l}{$\mathrm{PC} \%$} \\
\cline { 2 - 3 } \cline { 5 - 6 } & $I_{\text {lim }} \exp (\mathrm{A}) *$ & $I_{\text {lim }}$ calc $(\mathrm{A}) *$ & & $I_{\text {lim }} \exp (\mathrm{A}) *$ & $I_{\text {lim }}$ calc $(\mathrm{A}) *$ \\
$10^{-7}$ & $10^{-7}$ & & $10^{-7}$ & $10^{-7}$ \\
\hline 0 & - & 2.263 & & 1.103 & 1.379 \\
2 & - & 2.263 & & - & - \\
4 & 2.191 & 2.263 & & 1.370 & 1.450 \\
8 & - & - & 1.137 & 1.523 \\
\hline
\end{tabular}

\subsection{NEAs functionalization}

A fluorescent image of a functionalized array is shown in Fig. 4. The rectangles exhibiting higher fluorescent signal correspond to the areas observed by SEM before the process of functionalization. The exact mechanism leading to an increased density of probes immobilized in these areas has not been investigated in detail, but our hypothesis is that the energetic electron beam induces damage of the polymeric chains, which, after the exposure to air, lead to the formation of additional reactive sites, likely carboxylic groups, at the surface.

However the fluorescence signal that can be observed around the nanoelectrodes indicates the successful immobilization of the oligonucleotidic probes in proximity of the nanoelectrodes. A negative control, in order to assess whether the fluorescence is effectively related to the nucleotidic probes immobilization, has been done by treating an array with acD1 fluorescent probe with- 


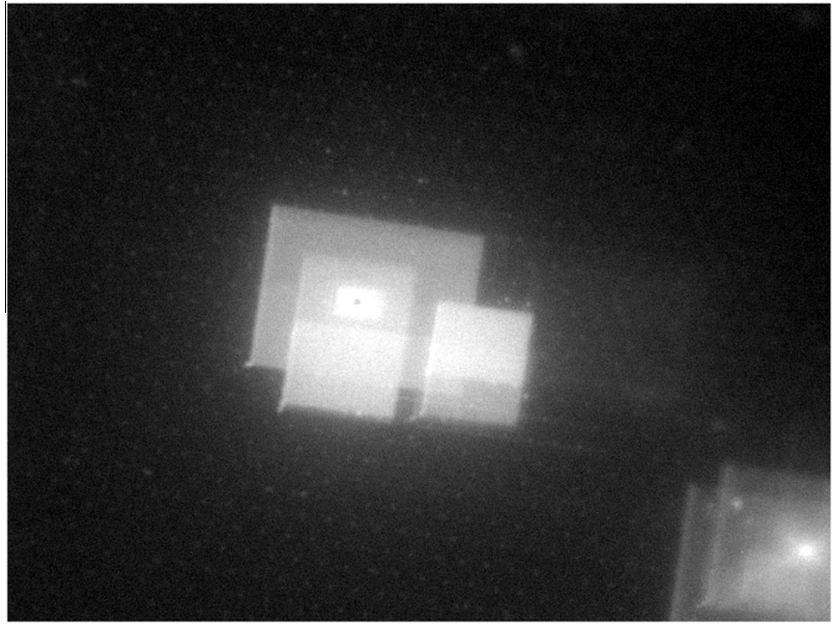

Fig. 4. Fluorescence microscope picture of functionalized NEA.

out the steps of chemical and plasma activation. In this case no fluorescence was observed in areas exposed and unexposed to the electron beam during the lithographic step, nor in the areas previously observed by SEM. This indicates the absence of aspecific binding of DNA on the surface, irrespective of whether the surface had been or not irradiated.

\section{Conclusions}

Electron Beam Lithography parameters for polycarbonate nanopatterning have been optimized and electrochemical behavior of our NEAs has been tested. The treatment with $\mathrm{O}_{2}$ plasma removes the residues of polycarbonate from the bottom of nanoelectrodes with consequent improvement of the voltammetric signals which acquire a sigmoidal shape over a broad range of scan rates, indicating that under these conditions the diffusion regime is pure radial (maximum improvement of signal to noise ratio). Experimental values of limiting current are in good agreement with the calculated ones. Moreover plasma treatment affects also the surface of polycarbonate, introducing oxygenated groups, useful for PC functionalization. The polycarbonate surface of the sensors has been successfully functionalized with small ss-DNA sequences, even if some aspects of the mechanism of immobilization and of surface activation are under evaluation.

\section{Acknowledgement}

We thank Dr. Morena Silvestrini for helpful discussions in DNA functionalization protocols.

\section{References}

[1] A.J. Cunningham, Introduction to Bioanalytical Sensors, J. Wiley \& Sons, New York, 1998.

[2] M. Zamuner, S. Pozzi Mucelli, M. Tormen, G. Stanta, P. Ugo, Eur. J. Nanomed. 1 (2008) 33.

[3] H.B. Shi, J.I. Yeh, Nanomedicine 2 (2007) 587-598.

[4] P. Ugo, L.M. Moretto, S. Bellomi, V.P. Menon, C.R. Martin, Anal. Chem 68 (1996) 169.

[5] V.P. Menon, C.R. Martin, Anal. Chem. 67 (1995) 1920

[6] C. Amatore, J.M. Saveant, D. Tessier, J. Electroanal. Chem. 147 (1983) 39.

[7] E. Sabatani, J. Rubinstein, J. Phys. Chem. 91 (1987) 6663.

[8] J. Guo, E. Lindner, Anal. Chem. 81 (2009) 130-138.

[9] M.E. Sandison, M. Cooper, Lab Chip 6 (2006) 1020-1025.

[10] L.M. Moretto, S. Panero, B. Scrosati, P. Ugo, in: Y. Lin, H.S. Nalwa (Eds.) Handbook of Electrochemical Nanotechnology, American Scientific Publishers, 2009, pp. 87-105.

[11] C. Prado, G.U. Flechsig, P. Gründler, J.S. Foord, F. Marken, R.G. Compton, Analyst 127 (2002) 329-332.

[12] R.V. Parhasarathy, C.R. Martin, Nature 396 (1994) 298.

[13] S. Pozzi Mucelli, M. Zamuner, M. Tormen, G. Stanta, P. Ugo, Biosens. Bioelectron. 22 (2008) 1900-1903.

[14] A. Carpentiero, M. De Leo, I.G. Romero, S. Pozzi Mucelli, F. Reuther, G. Stanta M. Tormen, P. Ugo, M. Zamuner, Nanoelectrochemical Immunosensors for Protein Detection, in: A. Schmid et al. (Eds.), Nano-Net 2009, LNICST 20, 2009, pp. $178-188$.

[15] L.M. Moretto, M. Tormen, M. De Leo, A. Carpentiero, P. Ugo, Nanotechnology 22 (2011) 185305.

[16] G. Pecchielan, Master Thesis, University Cà Foscari of Venice, 2010.

[17] A.M. Bond, D. Luscombe, K.B. Oldham, C.G. Zoski, J. Electroanal. Chem. 249 (1988) $1-14$. 